\title{
Cancer-associated retinopathy in patients with breast carcinoma
}

\section{Marta Misiuk-Hojło ${ }^{1}$, Maria Ejma², Wojciech A. Gorczyca ${ }^{3}$, Stanisław Szymaniec ${ }^{3}$, Danuta Witkowska ${ }^{3}$, Wojciech Fortuna ${ }^{3}$, Ryszard Międzybrodzki ${ }^{3}$, Jadwiga Rogozińska-Szczepka ${ }^{4}$ and Wiesława Bartnik ${ }^{4}$}

1 Department of Ophthalmology, Wrocław Medical University, Poland

2 Department of Neurology, Wrocław Medical University, Poland

3 Institute of Immunology and Experimental Therapy, Polish Academy of Sciences, Wrocław, Poland

4 Institute of Oncology, Gliwice, Poland

Received: 2007.02.02 Accepted: 2007.05.28, Published online first: 2007.07.23

\begin{abstract}
Introduction: Cancer-associated retinopathy (CAR) is a paraneoplastic neurological syndrome resulting in progressive loss of vision and clinical signs of retinal degeneration. It is associated with various types of cancer and is also considered to be an autoimmune disorder that involves cross-reaction between autoantibodies and retinal proteins. The aim of this study was to establish whether immunoreactivity to retinal antigens (RAs) observed in patients with breast cancer is accompanied by any visual impairments.

Materials and Methods: Sera of 295 patients with diagnosed breast cancer were screened for the presence of anti-RAs antibodies using immunoblotting. Cellular immunoreactivity to RAs present in retinal extracts and to purified recoverin and arrestin was determined by means of a lymphocyte proliferation assay. Six patients with high-titer antibodies to RAs then underwent ophthalmic and neurological examinations.

Results: Four serum samples contained high-titer antibodies to a 46-kDa protein, most probably retinal $\alpha$-enolase, three had antibodies to a 48-kDa protein identified as retinal arrestin, while 56-, 43-, 41-, and 34-kDa antigens were recognized only by one serum sample each. Moreover, weak cellular response to all the RAs tested was observed in one patient and another patient responded only to retinal extract. Two of the examined patients displayed symptoms of CAR.

Conclusions: Immunoreactivity to RAs in patients with breast cancer may also be present in cases without clinical signs of CAR.

Key words: retinal antigens, autoantibodies, breast cancer, visual disorders.

Abbreviations: CAR - cancer-associated retinopathy, cpm - counts per minute, ERG - electroretinogram, NBT/BCIP - nitro blue tetrazolium chloride/5-bromo-4-chloro-3-indolyl phosphate, toluidine salt, PNS - paraneoplastic neurological syndrome, RAs - retinal antigens, SCLC - small-cell lung cancer, SDS-PAGE - sodium dodecyl sulfate polyacrylamide gel electrophoresis, SI - stimulation index, VEP - visual evoked potentials.
\end{abstract}

Corresponding author: Marta Misiuk-Hojło, M.D. Ph.D., Department of Ophthalmology, Wrocław Medical University, Chałubińskiego 2a, 50-368 Wrocław, Poland, tel.: +48 71 784-24-27, fax: +48 71 784-15-83, e-mail: misiuk55@wp.pl

\section{INTRODUCTION}

Cancer-associated retinopathy (CAR) is a paraneoplastic neurological syndrome (PNS) resulting in progressive loss of vision and clinical signs of retinal degeneration. It is associated with various types of cancer, including small-cell lung cancer (SCLC), non-SCLC, breast cancer, invasive thymoma, uterine cervical cancer, endometrial cancer, lymphoma, and others $[15,16$, $25,27]$. The primary manifestation of CAR is rapid, progressive visual loss due to both rod and cone dysfunc- tion. Clinical problems associated with cone dysfunction include photosensitivity, decreased visual acuity, color vision abnormalities as well as central scotomas, and an abnormal cone-mediated electroretinogram (ERG). Clinical problems associated with rod dysfunction include nyctalopia, prolonged dark adaptation, peripheral or ring scotomas, and an abnormal rod-mediated ERG [19, 28]. The results of ophthalmologic examination often show a triad of symptoms: photosensitivity, ring scotomatous visual field loss, and attenuated retinal arteriole caliber [12]. Fundus findings in patients are not 
specific and could reveal normal fundus results, optic disc pallor, narrowing of the retinal arterioles, and retinal pigment epithelium changes [14, 17, 19, 28].

Similarly to other PNS, CAR is considered to be an autoimmune disorder that involves cross-reaction between autoantibodies and retinal proteins. Besides antibodies, cytotoxic cells may also mediate paraneoplastic-associated symptoms. Sera of patients with CAR have been shown to contain antibodies to several distinct retinal antigens (RAs), of which antibodies to a 23$-\mathrm{kDa}$ protein, identified as recoverin, appear to be the most common [21], but antibodies to $\alpha$-enolase [1, 3] and 34-, 43-, 60-, 65-, and 70-kDa proteins have also been detected [2, 9, 20, 24, 26]. Antibodies to some of these RAs have also been found in other neurological diseases [6]. On the other hand, it has also been reported that the presence of antibodies against RAs is not always accompanied by ocular symptoms [13, 23]. In this context, the aim of our study was to evaluate whether breast cancer patients whose sera or immunocompetent cells recognize RAs display any visual impairments.

\section{MATERIALS AND METHODS}

Serum samples were obtained from 295 patients diagnosed with breast cancer at the Institute of Oncology, Gliwice, Poland. The selection time period was six months. The mean interval time from the cancer onset and the study examination was from 6-12 months (mean 7.5 months). The sera were diluted 1:100 (standard dilution) and evaluated for the presence of antibodies against soluble RAs using immunoblot analysis. Six patients whose sera revealed high-titer antibodies to RAs (sera reactive at a dilution of at least 1:200) underwent ophthalmic and neurologic examinations. Their response to RAs at the cellular level was also evaluated. In all patients the presence of neoplasms of the central nervous system and concurrent eye conditions affecting the retina were excluded. They had no preceding illness (cardiovascular, hematologic nor metabolic disorders), trauma or toxin exposure. Non of them had a history of drugs known to influence visual function (two of them had received tamoxifen but after our examination). No family history of retinal degeneration existed. All assays and examinations were performed with the patients' consent and in accordance with institutional review board approval.

A complete ophthalmological examination was performed which included measurements of visual acuity, electroretinography (ERG), visual evoked potentials (VEP), intraocular pressure, indirect ophthalmoscopy using a Volk lens, and visual field by static perimetry. Best corrected visual acuity was measure using Snellen's scale. The ERG recordings (flash ERG and the $30 \mathrm{~Hz}$ flicker ERG) were performed using Tomey P400 device. Visual field was examined by means of an Octopus 101 perimeter. We performed the perimetric examination twice, according to the ST program and TOP strategy.
VEPs were investigated with use of averaging system manufactured by Nicolet (Nicolet CA-1000 averager).

To examine the reactivity of the sera in immunoblotting as well as the response at the cellular level, soluble RAs present in retinal extract were used. Retinal extract and purified RAs were obtained from dark-adapted bovine retinas according to procedures described earlier [13]. Briefly, the retinas were homogenized under dim red light in cold $\mathrm{H}_{2} \mathrm{O}$ containing $0.5 \mathrm{mM}$ benzamidine and $20 \mu \mathrm{g} / \mathrm{ml}$ of leupeptin (Sigma, St. Louis, MO, USA). The homogenate was centrifuged at $30,000 \times \mathrm{g}$ for $30 \mathrm{~min}$ at $4{ }^{\circ} \mathrm{C}$ and supernatant containing soluble proteins (the retinal extract) was collected and further used as a source of RAs. Arrestin and recoverin were purified from the retinal extracts by means of ion-exchange and Phenyl-Sepharose chromatographies, respectively.

Immunoblotting analysis was performed as follows: retinal extracts ( $10 \mu \mathrm{g}$ of proteins) or purified RAs (1.0 $\mu \mathrm{g})$ were resolved in SDS-PAGE using $12 \%$ acrylamide gels and transferred onto nitrocellulose membranes (Schleicher \& Schuell, Dassel, Germany). The membranes were blocked with $2 \%$ casein and incubated with samples of analyzed sera diluted 1:100 and 1:200 and then with anti-human IgG goat antibodies conjugated to alkaline phosphatase (Promega, Madison, WI, USA). Antibodies bound to RAs were visualized using NBT/BCIP-based substrate (Promega, Madison, WI, USA). The molecular masses of the detected proteins were determined using a Vilber Lourmat GelDoc system and BIO-CAPT and BIO-1D ${ }^{++}$software.

The cellular immune response to RAs, either purified (recoverin and arrestin) or those present in the retinal extract, was determined using a lymphocyte proliferation assay. Mononuclear cells were isolated from heparinized peripheral blood by centrifugation in density gradient using Histopaque 1077 (Sigma, St. Louis, MO, USA) and cultured with or without RAs (10-40 $\mu \mathrm{g} / \mathrm{ml}$ recoverin, arrestin, or retinal extract). Their proliferative responses were determined by measuring the incorporation of $\left[{ }^{3} \mathrm{H}\right]$-thymidine $(0.5 \mu \mathrm{Ci}$; Amersham Pharmacia Biotech, Uppsala, Sweden). The results are expressed in the form of a stimulation index (SI), calculated as the ratio of mean counts per minute (cpm) obtained from the cells stimulated with RAs and cpm obtained from unstimulated control cells. In accordance with reference [29], stimulation was considered positive if SI was equal to or greater than 2.

\section{RESULTS}

The reactivity of the patients' sera with RAs is presented in Fig. 1. Sera of four of the six patients (nos. 2, $3,4,6)$ contained high-titer antibodies (dilution above $1: 200)$ to a $46-\mathrm{kDa}$ protein, most probably retinal $\alpha$-enolase, three (nos. $1,5,6)$ had antibodies to a 48 -kDa protein identified as retinal arrestin, while 56-, 43-, 41-, and $34-\mathrm{kDa}$ antigens were recognized by one serum sample each (nos. $4,5,3$, and 1 , respectively). 


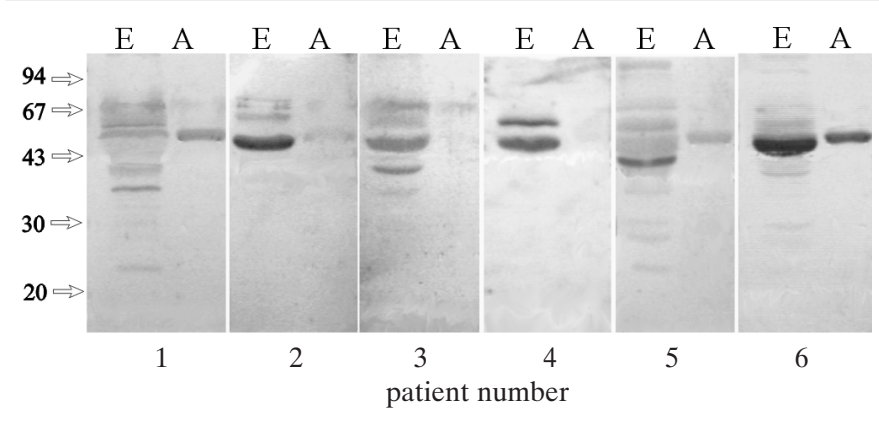

Fig. 1. Immunostaining of retinal antigens with sera of breast cancer patients. Lanes labeled (E) or (A) contain retinal extract or purified RAs (arrestin, recoverin, and guanylate cyclase-activating proteins), respectively. Antigens were immunostained with patients' sera diluted 1:200. Arrows indicate the positions of molecular mass markers.
The peripheral blood lymphocytes of one patient (no. 1) responded to retinal extract and to purified RAs (mean $\mathrm{SI} \geq 2$ ) and the lymphocytes of another (no. 3) responded exclusively to retinal extract. The cells of the remaining patients did not respond to any of the RAs tested (Table 1).

There were no neurological symptoms in three (nos. $1,5,6)$ of the six patients. In the other three (nos. 2, 3, 4) we found mild peripheral neuropathy (weak knee and ankle reflex, peripheral hypoesthesia of the lower extremities). The results of ophthalmological examination are shown in Table 2. All the patients had normal intraocular pressure. Although all of them displayed abnormal VEP, only two (nos. 1 and 2) showed abnormalities in ophthalmoscopy, visual field, and ERG. One of these patients (no. 2) revealed high-titer serum antibodies to the $46-\mathrm{kDa}$ antigen, while the serum of the other (no. 1) showed only moderate reactivity with RAs. Interestingly, only this patient's serum recognized a 34-kDa antigen. Cellular response to RAs was also most significant in this patient's lymphocytes (Table 1).

\section{DISCUSSION}

CAR has been increasingly diagnosed during the 29 years since Sawyer's discovery of it [22]. CAR always has to be taken into the consideration when confronted with a patient suffering from cancer and complaining of

Table 1. Proliferation of blood mononuclear cells (BMCs) from patients with breast cancer in response to retinal antigens

\begin{tabular}{cccc}
\hline \multirow{2}{*}{ Patient no. } & \multicolumn{3}{c}{ Antigen } \\
\cline { 2 - 4 } & E & Rcv & Ar \\
\hline 1 & $2.22 \pm 0.21$ & $2.09 \pm 0.37$ & $2.02 \pm 0.58$ \\
2 & - & - & - \\
3 & $2.16 \pm 0.12$ & - & - \\
4 & $1.42 \pm 0.24$ & $1.56 \pm 0.11$ & - \\
5 & - & - & - \\
6 & - & - & - \\
\hline
\end{tabular}

BMCs were stimulated with retinal extracts (E), recoverin ( $R c v)$, and arrestin (Ar) and then their proliferative responses were determined as described in Materials and Methods. Results obtained from at least three independent measurements are expressed as a mean value of the stimulation index $\pm \mathrm{SE}$. "dimming" of vision with missing areas of visual field, central and cecocentral scotomas, hemeralopia, photopsias, night blindness, loss of color vision, and photosensitivity. Laboratory examination for CAR in such a case is necessary. In our study group, a progressive decrease in visual function appeared in two patients in the course of six months and was bilateral. These signs and symptoms correlated with immunoreactivity to RAs, and the presence of breast cancer supported the diagnosis of CAR.

CAR symptoms may be observed before the diagnosis of cancer, as described in cases of SCLC, invasive thymoma, and uterine cervical cancer [2, 8, 14], but it has also been recognized after detecting a primary malignancy. An example of CAR symptoms developing after carcinoma was the case of a 65 -year-old man who underwent resection of adenocarcinoma of the lung. He noticed a deterioration of vision 10 months later [19]. With regard to patients with breast cancer, two patients have been reported who both presented paraneoplastic retinopathy symptoms although their sera did not contain antibodies to the most common RA in CAR, namely recoverin [11]. In our patients the CAR symptoms developed after the diagnosis of cancer and the lymphocytes of only one of them responded weakly to recoverin. However, their sera contained antibodies to the 46and 34-kDa proteins also detected in CAR patients with SCLC [3] and endometrial carcinoma [24]. It has been reported that breast cancer may also be associated with cerebellar degeneration, sensoriomotor neuropathy, and opsoclonus/myoclonus syndrome, which all represent PNSs other than CAR [5, 7]. In our three patients we found mild peripheral neuropathy, which together with other impairments (VEP pathology) may also indicate a PNS.

In conclusion, this study showed that antibodies to RAs are present in the sera of patients with breast cancer. However, their presence correlated only in few cases with the visual impairments characteristic for CAR symptoms. Reactivity to RAs of sera in patients with breast cancer could also be present in cases without clinical signs of CAR. However, detecting and monitoring the presence of anti-RAs antibodies as well as their titers with parallel neurological and ophthalmic examinations can be useful in patient management and give an insight into the relations between a cancer and the development of neurological and ophthalmic symptoms. 
Table 2. Summary of patients

\begin{tabular}{|c|c|c|c|c|c|c|c|}
\hline $\begin{array}{c}\text { Patient } \\
\text { no. }\end{array}$ & $\begin{array}{c}\text { Age (years), } \\
\text { gender } \\
(\mathrm{R} ; \mathrm{L})\end{array}$ & $\begin{array}{c}\text { VEP } \\
\text { latency }(\mathrm{ms}) \\
(\mathrm{R} ; \mathrm{L})\end{array}$ & $\begin{array}{l}\text { Visual } \\
\text { acuity } \\
(\mathrm{R} ; \mathrm{L})\end{array}$ & $\begin{array}{l}\text { Visual } \\
\text { fields }\end{array}$ & Ophthalmoscopy & ERG & $\begin{array}{c}\text { Main RA } \\
\text { detected }\end{array}$ \\
\hline 1 & $47, \mathrm{~F}$ & $120 ; 120$ & $0.2 ; 0.1$ & A & arteriolar narrowing & reduced & arrestin; p34 \\
\hline 2 & $54, \mathrm{~F}$ & $120 ; 108$ & $0.02 ; 0.1$ & $\mathrm{~B}$ & arteriolar narrowing & reduced & $\mathrm{p} 46$ \\
\hline 3 & $54, \mathrm{~F}$ & $122 ; 130$ & $0.9 ; 1.0$ & normal & normal & normal & $\mathrm{p} 46 ; \mathrm{p} 41$ \\
\hline 4 & $62, \mathrm{~F}$ & $116 ; 118$ & $0.8 ; 1.0$ & normal & normal & normal & p56; p46 \\
\hline 5 & $69, \mathrm{~F}$ & $124 ; 120$ & $0.7 ; 0.4$ & normal & normal & normal & arrestin; p43 \\
\hline 6 & $65, \mathrm{~F}$ & $120 ; 122$ & $1.0 ; 1.0$ & normal & normal & normal & arrestin; $\mathrm{p} 46$ \\
\hline
\end{tabular}

$\mathrm{R}$ - right eye, $\mathrm{L}$ - left eye, $\mathrm{ms}$ - milliseconds, $\mu \mathrm{V}$ - microvolt, VEP - visual evoked potentials; the latencies above $115.5 \mathrm{~ms}$ were considered "abnormal", following our previous studies in a normal population [4], ERG - electroretinogram; amplitudes and peak times (30 Hz flicker ERG) below $115.20 \pm 60.74 \mu \mathrm{V}$ and $20.14 \pm 4.12 \mathrm{~ms}$ were considered "reduced" following our previous studies in a normal population (not reported) and other author's studies [18].

Visual field was considered normal if extends to 35 degrees nasally, to 60 degrees temporally, 50 degrees above and below the horizontal meridian, the macula corresponds to the central 13 degrees. A - ring scotomatous visual field loss, $\mathrm{B}-$ constriction to $25-30^{\circ}$ in the right eye; temporal constriction to $20^{\circ}$ in the left eye.

Ophthalmoscopy was considered normal if the retina, blood vessels, and the optic disc appear normal to the examiner.

Arterioles were considered narrowing according to the protocol used in the ARIC study [10].

Acknowledgement: This study was supported by grant no. 4 P05A 08214 from the State Committee for Scientific Research (KBN, Poland).

\section{REFERENCES}

1. Adamus G., Aptsiauri N., Guy J., Heckenlively J., Flannery J. and Hargrave P. A. (1996): The occurrence serum autoantibodies against enolase in cancer-associated retinopathy. Clin. Immunol. Immunopathol., 78, 120-129.

2. De Potter D. (1998): Ocular manifestation of cancer. Curr. Opin. Ophthalmol., 9, 100-104.

3. Dot C., Guigay J. and Adamus G. (2005): Anti- $\alpha$-enolase antibodies in cancer-associated retinopathy with small cell carcinoma of the lung. Am. J. Ophthalmol., 139, 746-747.

4. Ejma M., Bieszczad M., Podemski R., Bilińska M., Usnarska-Zubkiewicz L. (1995): Wzrokowe i somatosensoryczne potencjały wywołane u chorych $\mathrm{z}$ chłoniakiem nieziarniczym. Neurol. Neurochir. Pol., 29, 353-362.

5. Gatti G., Simsek S., Kurne A., Zurrida S., Naninato P., Veronesi P., Frasson A., Millen E., Rososchansky J. and Luini A. (2003): Paraneoplastic disorders in breast cancer. Breast, 12, 203-207.

6. Gorczyca W., A., Ejma M., Witkowska D., Misiuk-Hojlo M., Kuropatwa M., Mulak M. and Szymaniec S. (2004): Retinal antigens are recognized by antibodies present in sera of patients with multiple sclerosis. Ophthalmic Res., 36, 120-123.

7. Graus F., Delattre J. Y., Antoine J. C., Dalmau J., Giometto B., Grisold W., Honnorat J., Sillevis Smitt P., Vedeler Ch., Verschuuren J. J. G. M., Vincent A. and Voltz R. (2004): Recommended diagnostic criteria for paraneoplastic neurological syndromes. J. Neurol. Neurosurg. Psych., 75, 1135-1140.

8. Grunwald G., B., Klein R., Simmonds M. A. and Kornguth S. E. (1985): Autoimmune basis for visual paraneoplastic syndrome in patients with small-cell lung carcinoma. Lancet, 1, 658-661.
9. Hooks J. J., Tso M. O. and Detrick B. (2001): Retinopathies associated with antiretinal antibodies. Clin. Diagn. Lab. Immunol., 8, 853-858.

10. Hubbard L. D., Brothers R. J., King W. N., Clegg L. X., Klein R., Cooper L. S., Sharrett A. R., Davis M. D. and Cai J. (1999): Methods for evaluation of retinal microvascular abnormalities associated with hypertension/sclerosis in the Atherosclerosis Risk in Communities Study. Ophthalmology, 106, 2269-2280.

11. Huober J., Holz F. G., Schmid H., Nolle B., Bellmann C., Krastel H., Wallwiener D. and Bastert G. (1997): Paraneoplastic retinopathy in 2 patients with breast carcinoma. Zentralbl. Gynakol., 119, 278-281.

12. Jacobson D. M., Thirkill C. E. and Tipping S. J. (1990): A clinical triad to diagnose paraneoplstic retinopathy. Ann. Neurol., 28, 162-167.

13. Jankowska R., Witkowska D., Porębska I., Kuropatwa M., Kurowska E. and Gorczyca W. A. (2004): Serum antibodies to retinal antigens in lung cancer and sarcoidosis. Pathobiology, 71, 323-328.

14. Katsuta H., Okada M., Nakauchi T., Takahashi Y., Yamao S. and Uchida S. (2002): Cancer-associated retinopathy with invasive thymoma. Am. J. Ophthalmol., 134, 383-389.

15. Keltner J. L., Thirkill C. E. and Yip P. T. (2001): Clinical and immunologic characteristics of melanoma-associated retinopathy syndrome: eleven new cases and a review of 51 previously published cases. J. Neuroophthalmol., 21, 173-187.

16. Misiuk-Hojło M., Jurowska-Liput J. and Gorczyca W. (2004): Cancer associated retinopathy. Nowotwory J. Oncol., 54, 584-586.

17. Murphy M. A., Thirkill C. E. and Hart W. M. Jr. (1997): Paraneoplastic retinopathy: a novel autoantibody reaction associated with small-cell lung carcinoma. J. Neuroophthalmol., 17, 77-83.

18. Nordenfeld L. and Ohman R. (1983): Ruotine ERG recording using medium frequency flicker stimulus. Acta Ophthalmol., 61, 29-37.

19. Ohguro H., Maruyama I., Nakazawa M. and Oohira A. (2002): Antirecoverin antibody in the aqueosus humor of 
patient with cancer- associated retinopathy. Am. J. Ophthalmol., 134, 605-607.

20. Ohguro H., Ogawa K. and Nakagawa T. (1999): Recoverin and Hsc 70 are found as autoantigens in patients with cancer-associated retinopathy. Invest. Ophthalmol. Vis. Sci., 40, 82-89.

21. Ohguro H., Yokoi Y., Ohguro I., Mamiya K., Ishikawa F., Yamazaki H., Metoki T., Takano Y., Ito T. and Nakazawa M. (2004): Clinical and immunologic aspects of cancerassociated retinopathy. Am. J. Ophthalmol., 137, 1117-1119.

22. Sawyer R. A., Selhorst J. B., Zimmerman L. E. and Hoyt W. F. (1976): Blindness caused by photoreceptor degeneration as a remote effect of cancer. Am. J. Ophthalmol., 81, 606-613.

23. Savchenko M. S., Bazhin A. V., Shifrina O. N., Demoura S. A., Kogan E. A., Chuchalin A. G. and Philippov P. P. (2003): Antirecoverin autoantibodies in the patient with non-small cell lung cancer but without cancer-associated retinopathy. Lung Cancer, 41, 363-367.

24. Sekiguchi I., Suzuki M., Sato I., Ohkawa T., Kawashima H. and Tsuchida S. (1998): Rare case of small-cell carcinoma arising from the endometrium with paraneoplastic retinopathy. Gynecol. Oncol., 71, 454-457.

25. Suhler E. B., Chan C. C., Caruso R. C., Schrump D. S., Thirkill C., Smith J. A., Nussenblatt R. B. and Buggage R. R. (2003): Presumed teratoma-associated paraneoplastic retinopathy. Arch. Ophthalmol., 121, 133-137.

26. Thirkill C. E., Fitzgerald P., Sergott R. C., Roth A. M., Tyler N. K. and Keltner J. L. (1989): Cancer-associated retinopathy (CAR syndrome) with antibodies reacting with retinal, optic nerve, and cancer cells. N. Engl. J. Med., 321, 1589-1594.

27. To K. W., Thirkill C. E., Jakobiec F. A., Lessel S. and Berson E. L. (2002): Lymphoma-associated retinopathy. Ophthalmology, 109, 2149-2153.

28. Whitcup S. M., Vistica B. P., Milam A. H., Nussenblatt R. B. and Gery I. (1998): Recoverin-associated retinopathy: a clinically and immunologically distinctive disease. Am. J. Ophthalmol., 126, 230-237.

29. Zhang S., Jiang S., Manczak M., Sugden B. and Adamus A. (1998): Phenotypes of $\mathrm{T}$ cells infiltrating the eyes in autoimmune anterior uveitis associated with EAE. Invest. Ophthalm. Vis. Sci., 43, 1499-1508. 
